\title{
Wave Propagation in Networks: a System Theoretic Approach *
}

\author{
Atte Aalto and Jarmo Malinen
}

\author{
Department of Mathematics and Systems Analysis, \\ Aalto University School of Science, Espoo, Finland \\ (e-mail: aaalto@cc.hut.fi and jmalinen@cc.hut.fi).
}

\begin{abstract}
We consider dynamical systems defined on graph structures. The dynamics on the edges are governed by partial differential equations that are interconnected at the graph vertices through algebraic conditions involving the boundary conditions of the PDEs. We show that a variety of such wave propagation problems on networks are solvable (forward in time) and energy passive or conservative - given that the governing PDEs are solvable on the separate edges. We treat these problems in the operator theoretic boundary control system framework.
\end{abstract}

Keywords: Distributed parameter systems, Mathematical systems theory, Nyquist's criterion, Wave guides, Boundary control systems, Coupled system

\section{INTRODUCTION}

Wave propagation problems in networks arise in many fields of technology. Let us give a brief list of possible applications with references:

- Acoustics: see, e.g., Hannukainen et al. (2007) studying the acoustics of the human vocal tract that can be considered as a Y-shaped graph;

- Mechanics: see, e.g., Mei and Mace (2005) where the wave propagation problem in Timoshenko beam structures is studied. In particular, the jointed beams and beams with parameter discontinuities can be regarded as network systems;

- Electrodynamics: see, e.g., Gorbachuk and Gorbachuk (1991); Malinen and Staffans (2007) where the transmission line equations are formulated as a boundary control system.

In this paper we show that a variety of wave propagation problems on networks are solvable (forward in time) and energy passive or conservative. These problems are given in terms of partial differential equations that are interconnected through certain algebraic conditions involving the boundary conditions of these PDEs. The contribution of this paper is to show that if all of the single PDEs are solvable and energy passive (or conservative) then also the interconnected PDE-system is solvable and energy passive (respectively, conservative).

In more technical terms, we formulate the subsystems of the network as distributed parameter boundary control systems (BCS) that are an operator theoretic framework for treating problems concerning PDEs to which control action is inflicted through boundary conditions. In this framework, the interconnections between the subsystems become algebraic conditions in terms of the subsystems' input and output operators, of which we only require

\footnotetext{
^ A. Aalto was financially supported by the Finnish graduate school in engineering mechanics.
}

that they are of compatible dimensions. We then show that the interconnections can be constructed so that the composed systems are always solvable and energy passive/conservative.

The background on boundary control systems is covered in Section 2. The concept of a transmission graph, that is, a composition of BCSs, is presented in Section 3. In Section 4, we present three elementary operations, by which it is possible to synthesise any transmission graph structure from more simple subsystems. These elementary operations preserve solvability and energy (in)equalities - also when applied iteratively, see Theorem 6 , the main result of this paper. This theorem can also be regarded as Nyquist's criterion for passive boundary control systems: any composition of passive BCSs is also a passive BCS.

Our results are illustrated by Webster's equation on a simple graph shown in Fig. 1. Next, in Section 1.1, we shall formulate a subsystem of this structure (that is, Webster's equation on a line) as a boundary control system. The example is revisited in Section 4.4 where we first formulate the composed system as a transmission graph and then show how this example graph is reconstructed using the three elementary operations.

All the proofs in this paper are only briefly outlined. For complete proofs, we refer to Aalto and Malinen (2010), as well as for details of our example. Our approach is based on results of Malinen and Staffans (2006, 2007) on boundary control systems. Other related work includes Staffans (2005) and Weiss (1994) who study the feedback theory for (regular) well-posed linear systems. Similar approaches for composing conservative linear systems can be found in Villegas (2007), Cervera et al. (2007), Kurula et al. (2010), and Derkach et al. (2006).

\subsection{Webster's equation on a graph}

Let us consider the following example from acoustic wave propagation. Given the interconnection graph in Fig. 1, 
the longitudinal wave propagation on its edges (i.e., wave guides) is described by the dynamics of the velocity potential $\psi^{(j)}$, governed by

$$
\frac{\partial^{2} \psi^{(j)}}{\partial t^{2}}(x, t)=\frac{c^{2}}{A_{j}(x)} \frac{\partial}{\partial x}\left(A_{j}(x) \frac{\partial \psi^{(j)}}{\partial x}(x, t)\right)
$$

where $t \in \mathbb{R}^{+}, x \in\left[0, L_{j}\right]$, and $L_{j}$ is the length of the wave guide. The distributed parameters $A_{j}(\cdot)>0$ can be understood as the cross-sectional areas of the wave guides. The index $j=A, \ldots, D$ refers to the index of the edge, and the arrows in Fig. 1 show the positive direction of the parametrisation $x \in\left[0, L_{j}\right]$. To the vertices $A B D$ and $B C D$ we impose Kirchhoff law type coupling (boundary) conditions (take vertex $A B D$ for example):

$$
\left\{\begin{array}{c}
\frac{\partial \psi^{(A)}}{\partial t}\left(L_{A}, t\right)=\frac{\partial \psi^{(B)}}{\partial t}(0, t)=\frac{\partial \psi^{(D)}}{\partial t}\left(L_{D}, t\right) \\
A_{A}\left(L_{A}\right) \frac{\partial \psi^{(A)}}{\partial x}\left(L_{A}, t\right)-A_{B}(0) \frac{\partial \psi^{(B)}}{\partial x}(0, t) \\
+A_{D}\left(L_{D}\right) \frac{\partial \psi^{(D)}}{\partial x}\left(L_{D}, t\right)=0 .
\end{array}\right.
$$

We remark that velocity potential gives the perturbation pressure and velocity through $p^{(j)}=\rho \frac{\partial \psi^{(j)}}{\partial t}$ and $v^{(j)}=$ $-\frac{\partial \psi^{(j)}}{\partial x}$, respectively. Thus, the first equation in (2) says that the pressure is continuous, and the second equation is a flow conservation law.

We want to control the pressure at the vertex $A C$ and observe the perturbation flux to the wave guides $A$ and $C$. Defining the input and output

$$
\left\{\begin{array}{l}
u(t):=\frac{\partial \psi^{(A)}}{\partial t}(0, t)=\frac{\partial \psi^{(C)}}{\partial t}(0, t), \\
y(t):=-A_{A}(0) \frac{\partial \psi^{(A)}}{\partial x}(0, t)-A_{C}(0) \frac{\partial \psi^{(C)}}{\partial x}(0, t),
\end{array}\right.
$$

respectively, then equations (1) for $j=A, \ldots, D$ and (2) define a dynamical system whose solvability and energy conservation we wish to verify using Theorem 6 below.

The contribution of this theorem is that it is only required to verify the solvability of the subsystems, that is, equations (1) on the edges with boundary conditions

$$
\left[\begin{array}{c}
\frac{\partial \psi^{(j)}}{\partial t}(0, t) \\
\frac{\partial \psi^{(j)}}{\partial t}\left(L_{j}, t\right)
\end{array}\right]=\left[\begin{array}{l}
u_{1}^{(j)}(t) \\
u_{2}^{(j)}(t)
\end{array}\right]=: u^{(j)}(t) .
$$

After reducing (1) to a first order equation of form $\dot{z}=L z$ with $z=\left[\begin{array}{l}\psi^{(j)} \\ p^{(j)}\end{array}\right]$, defining operator $G$ by $G z(t)=u^{(j)}(t)$, and $K$ in a similar manner, we obtain an internally wellposed boundary node $\Xi^{(j)}=(G, L, K)$ that is impedance conservative (see Definitions 2 and 3 ) and $\left[\begin{array}{c}G \\ K\end{array}\right]$ is surjective. As explained after Definition 2, the initial value problem

$$
\begin{aligned}
& u(t)=G z(t), \\
& \dot{z}(t)=L z(t), \quad \\
& y(t)=K z(t), \quad t \in \mathbb{R}^{+}, \\
& z(0)=z_{0}
\end{aligned}
$$

has a solution such that $\psi^{(j)}$ in equation (1) satisfies $\psi^{(j)} \in C^{1}\left(\mathbb{R}^{+}, L^{2}\left(0, L_{j}\right)\right) \cap C\left(\mathbb{R}^{+}, H^{1}\left(0, L_{j}\right)\right)$ for all inputs $u^{(j)} \in C^{2}\left(\mathbb{R}^{+}, \mathbb{C}^{2}\right)$ and for all initial states $z_{0}$ that satisfy the boundary condition (4), too. For technical details, see Malinen and Staffans (2007).

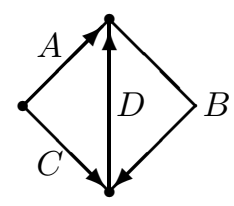

Fig. 1. The example graph

The boundary nodes $\Xi^{(j)}, j=A, \ldots, D$, together with coupling conditions of the form (2) for vertices $A B D$ and $B C D$, and external input and output through (3), form a transmission graph as defined in Definition 4. We shall conclude in Section 4.4 that this transmission graph gives rise to an impedance conservative boundary node and hence, the composed system is solvable forward in time and energy conservative.

\subsection{Notation and remarks}

In this paper we use the notations $[:]$ and $\bigoplus$ to represent orthogonal direct sum of (sub)spaces. By "=" between Hilbert spaces we mean unitary equivalence. The null space of an operator is denoted by $\mathcal{N}(\cdot)$.

\section{BOUNDARY CONTROL SYSTEMS}

We work in the abstract framework of boundary control systems described by operator differential equations of the form (5) involving linear mappings $G, L$, and $K$. For an introduction of such systems, see (Malinen and Staffans, 2006, Section 1) and (Malinen and Staffans, 2007, Section 2) and references therein.

Definition 1. Let $\Xi:=(G, L, K)$ be a triple of linear mappings.

(i) $\Xi$ is a colligation on the Hilbert spaces $(\mathcal{U}, \mathcal{X}, \mathcal{Y})$ if $G$, $L$, and $K$ have the same domain $\mathcal{Z}=\operatorname{dom}(\Xi) \subset \mathcal{X}$ and values in $\mathcal{U}, \mathcal{X}$, and $\mathcal{Y}$, respectively;

(ii) A colligation $\Xi$ is strong if $\left[\begin{array}{l}G \\ L \\ K\end{array}\right]$ is closed as an operator $\mathcal{X} \rightarrow\left[\begin{array}{l}\mathcal{U} \\ \mathcal{X} \\ \mathcal{Y}\end{array}\right]$ with domain $\mathcal{Z}$, and $L$ is closed with $\operatorname{dom}(L)=\mathcal{Z}$.

We call $L$ the interior operator, $G$ the input (boundary) operator, $K$ the output (boundary) operator. The space $\mathcal{Z}$ we call the solution space, $\mathcal{X}$ the state space, and $\mathcal{U}$ and $\mathcal{Y}$ the input and output spaces, respectively. In $\mathcal{Z}$ we use the graph norm $\|z\|_{\mathcal{Z}}^{2}:=\|z\|_{\mathcal{X}}^{2}+\|G z\|_{\mathcal{U}}^{2}+\|L z\|_{\mathcal{X}}^{2}+\|K z\|_{\mathcal{Y}}^{2}$.

Dynamical systems defined by boundary controlled partial differential equations naturally adopt the form (5) associated with some colligation $(G, L, K)$ on properly chosen spaces $(\mathcal{U}, \mathcal{X}, \mathcal{Y})$. Equations $(5)$ are solvable forward in time (at least) if $\Xi$ satisfies somewhat stronger assumptions:

Definition 2. A strong colligation $\Xi=(G, L, K)$ is a boundary node on the Hilbert spaces $(\mathcal{U}, \mathcal{X}, \mathcal{Y})$ if:

(i) $G$ is surjective and $\mathcal{N}(G)$ is dense in $\mathcal{X}$;

(ii) The operator $\left.L\right|_{\mathcal{N}(G)}$ (interpreted as an operator in $\mathcal{X}$ with domain $\mathcal{N}(G)$ ) has a nonempty resolvent set.

This boundary node is internally well-posed if, in addition,

(iii) $\left.L\right|_{\mathcal{N}(G)}$ generates a $C_{0}$ semigroup. 
This definition coincides with (Malinen and Staffans, 2006, Definition 1.1) for strong colligations. There are, in fact, well-posed boundary nodes that are not strong (see (Malinen and Staffans, 2007, Proposition 6.3)) but we do not consider such nodes in this paper ${ }^{1}$. We remark that also Fattorini (1968); Gorbachuk and Gorbachuk (1991); Kurula et al. (2010) treat strong colligations (with different names), see (Malinen and Staffans, 2007, Theorem 5.2) and (Kurula et al., 2010, Remark 4.4).

The term "boundary node" does not refer to the vertices of the underlying graph structure. In fact, boundary nodes are related to the edges of the graph. Therefore, we always talk about vertices when referring to the graph structure.

If $\Xi=(G, L, K)$ is an internally well-posed boundary node, then (5) has a unique solution for sufficiently smooth input functions $u$ and initial states $z_{0}$ compatible with $u(0)$. More precisely, as shown in (Malinen and Staffans, 2006, Lemma 2.6), for all $z_{0} \in \mathcal{Z}$ and $u \in C^{2}\left(\mathbb{R}^{+} ; \mathcal{U}\right)$ with $G z_{0}=u(0)$ the first, second, and fourth of the equations (5) have a unique solution $z \in C^{1}\left(\mathbb{R}^{+} ; \mathcal{X}\right) \cap C\left(\mathbb{R}^{+} ; \mathcal{Z}\right)$, and hence $y \in C\left(\mathbb{R}^{+} ; \mathcal{Y}\right)$ can be defined by the third equation in (5). In the rest of this article, by "a smooth solution of (5) on $\mathbb{R}^{+}$" we mean a solution with the above properties.

In a practical application, checking the solvability of (5) by verifying the conditions of Definition 2 may be difficult. However, in many cases this is not necessary because the system satisfies energy (in)equalities that can be verified using the Green-Lagrange inequality without an a priori knowledge of the well-posedness. Such energy laws are very useful when checking the solvability, see (Malinen and Staffans, 2007, Theorems 4.3 and 4.7). These two theorems are the most important tools in our proofs.

Next we shall define impedance passivity/conservativity. To keep the notation simple, we assume that $\mathcal{U}=\mathcal{Y}$ even though it would be enough to assume that $\mathcal{U}$ and $\mathcal{Y}$ are a dual pair of Hilbert spaces.

Definition 3. Let $\Xi=(G, L, K)$ be a colligation on Hilbert spaces $(\mathcal{U}, \mathcal{X}, \mathcal{Y})$

(i) $\Xi$ is impedance passive if the following conditions hold:

(a) $\left[\begin{array}{c}\beta G+K \\ \alpha-L\end{array}\right]$ is surjective for some $\alpha, \beta \in \mathbb{C}^{+}$;

(b) For all $z \in \operatorname{dom}(\Xi)$ we have the Green-Lagrange inequality

$$
\operatorname{Re}\langle z, L z\rangle_{\mathcal{X}} \leq \operatorname{Re}\langle K z, G z\rangle_{\mathcal{U}}
$$

(ii) Impedance passive $\Xi$ is impedance conservative if (6) holds as an equality, and (a) holds also for some $\alpha, \beta \in \mathbb{C}^{-}$.

The condition (a) stems from (Malinen and Staffans, 2007, Definition 3.2) where impedance passivity/conservativity is defined using the external Cayley transform of scattering passivity/conservativity (see also the discussion there). By (Malinen and Staffans, 2007, Theorem 3.4), this definition is equivalent with our Definition 3 above. This theorem also states that for an impedance passive $\Xi$, condition (a) holds for all $\alpha, \beta \in \mathbb{C}^{+}$, and for an impedance conservative $\Xi$, condition (a) holds also for all $\alpha, \beta \in \mathbb{C}^{-}$.

1 To avoid confusion, we use the term strong boundary node below.
Suppose now that $\Xi$ is an internally well-posed, impedance passive boundary node and $z$ a smooth solution of (5). Then (6) means plainly the energy inequality

$$
\frac{d}{d t} \frac{1}{2}\|z(t)\|_{\mathcal{X}}^{2} \leq \operatorname{Re}\langle y(t), u(t)\rangle_{\mathcal{U}} \quad \text { for all } t \in \mathbb{R}^{+}
$$

where the right hand side stands for the instantaneous power inflicting the system, and the norm of $\mathcal{X}$ measures the energy stored in the state.

\section{TRANSMISSION GRAPHS AS COLLIGATIONS}

Assume that $\Xi^{(j)}=\left(G^{(j)}, L^{(j)}, K^{(j)}\right)$ are colligations on Hilbert spaces $\left(\mathcal{U}^{(j)}, \mathcal{X}^{(j)}, \mathcal{Y}^{(j)}\right)$ with solution spaces $\mathcal{Z}^{(j)}, j=1, \ldots, m$, where

$$
G^{(j)}=\left[\begin{array}{lll}
G_{1}^{(j)} & \cdots & G_{k_{j}}^{(j)}
\end{array}\right]^{T}: \operatorname{dom}\left(\Xi^{(j)}\right) \rightarrow \mathcal{U}^{(j)}=\bigoplus_{i=1}^{k_{j}} \mathcal{U}_{i}^{(j)}
$$

and

$$
K^{(j)}=\left[\begin{array}{lll}
K_{1}^{(j)} & \cdots & K_{k_{j}}^{(j)}
\end{array}\right]^{T}: \operatorname{dom}\left(\Xi^{(j)}\right) \rightarrow \mathcal{Y}^{(j)}=\bigoplus_{i=1}^{k_{j}} \mathcal{Y}_{i}^{(j)} .
$$

That is, the Hilbert spaces $\mathcal{U}^{(j)}$ and $\mathcal{Y}^{(j)}$ are represented by an orthogonal direct sum of $k_{j}$ subspaces each, and the respective input and output operators are split accordingly. Following this splitting, we define the index set

$$
\text { Ind }:=\left\{(j, i) \in \mathbb{N} \times \mathbb{N} \mid j=1, \ldots, m ; i=1, \ldots, k_{j}\right\}
$$

and represent it as a union $\mathcal{I} n d=\bigcup_{k=1}^{N} \mathcal{I}^{k} \cup \bigcup_{l=1}^{M} \mathcal{J}^{l}$ of pairwise disjoint sets $\mathcal{I}^{1}, \ldots, \mathcal{I}^{N}$ and $\mathcal{J}^{1}, \ldots, \mathcal{J}^{M}$. Each of the sets $\mathcal{I}^{k}$ or $\mathcal{J}^{l}$ describes individual couplings of signals, and we name the sets control and closed vertices, respectively. They define a (hyper)graph structure where inputs and outputs of colligations $\Xi^{(j)}$ are coupled by algebraic equations (8) and (9) below. In order to make the couplings possible, it is required that the compatibility conditions

$$
\mathcal{U}_{i}^{(j)}=\mathcal{U}_{q}^{(p)} \text { and } \mathcal{Y}_{i}^{(j)}=\mathcal{Y}_{q}^{(p)}
$$

hold for all $(j, i),(p, q) \in \mathcal{I}^{k}, k=1, \ldots, N$ and for all $(j, i),(p, q) \in \mathcal{J}^{l}, l=1, \ldots, M$.

Definition 4. Assume that $\Xi^{(j)}$ are colligations with splittings as described above. Suppose that sets $\mathcal{I}^{1}, \ldots, \mathcal{I}^{N}, N \neq$ 0 and $\mathcal{J}^{1}, \ldots, \mathcal{J}^{M}$ are defined consistently with the splittings so that the compatibility conditions (7) hold.

The ordered triple

$$
\Gamma:=\left(\left\{\Xi^{(j)}\right\}_{j=1}^{m},\left\{\mathcal{I}^{k}\right\}_{k=1}^{N},\left\{\mathcal{J}^{l}\right\}_{l=1}^{M}\right)
$$

is a transmission graph with (Kirchhoff) couplings

(i) for all control and closed vertices, the continuity equations

$$
G_{i}^{(j)} z^{(j)}=G_{q}^{(p)} z^{(p)}, \quad z^{(j)} \in \mathcal{Z}^{(j)}, z^{(p)} \in \mathcal{Z}^{(p)}
$$

hold, i.e., (8) holds for all $(j, i),(p, q) \in \mathcal{I}^{k}, k=$ $1, \ldots, N$ and for all $(j, i),(p, q) \in \mathcal{J}^{l}, l=1, \ldots, M$; and

(ii) for closed vertices, the balance equations

$$
\sum_{(j, i) \in \mathcal{J}^{l}} K_{i}^{(j)} z^{(j)}=0, \quad z^{(j)} \in \mathcal{Z}^{(j)}, l=1, \ldots, M
$$


The control vertices are exactly those couplings where external signals are applied. If the transfer function (see (Malinen and Staffans, 2006, Section 2)) of each $\Xi^{(j)}$ represents electrical admittance, then the physical dimensions of $\mathcal{U}^{(j)}$ and $\mathcal{Y}^{(j)}$ are the voltage and current, respectively, and (8) and (9) are the classical Kirchhoff laws: the continuity of voltage and the conservation of charge.

Definition 5. Let $\Gamma$ be a transmission graph as in Definition 4 . Using the same notation, the colligation of the transmission graph is defined as the triple $\Xi_{\Gamma}=(G, L, K)$ on the Hilbert spaces $(\mathcal{U}, \mathcal{X}, \mathcal{Y})$ where ${ }^{2}$

$$
\begin{gathered}
\mathcal{X}:=\bigoplus_{j=1}^{m} \mathcal{X}^{(j)}, \quad \mathcal{U}:=\bigoplus_{\substack{(j, i) \in \mathcal{I}^{k} \\
k=1, \ldots, N}} \mathcal{U}_{i}^{(j)}, \quad \mathcal{Y}:=\bigoplus_{\substack{(j, i) \in \mathcal{I}^{k} \\
k=1, \ldots, N}} \mathcal{Y}_{i}^{(j)}, \\
\operatorname{dom}\left(\Xi_{\Gamma}\right):=\left\{\bigoplus_{j=1}^{m} \mathcal{Z}^{(j)} \mid(8) \text { and }(9) \text { hold }\right\}, \\
G:=\left[G_{k, j}\right]_{\substack{k=1, \ldots, N \\
j=1, \ldots, m}} L:=\left[\begin{array}{c}
L^{(1)} \\
\ddots \\
\text { and } K:=\left[K_{k, j}\right]_{\substack{k=1, \ldots, N \\
j=1, \ldots, m}}^{(m)}
\end{array}\right],
\end{gathered}
$$

where

$$
\begin{gathered}
G_{k, j}:= \begin{cases}G_{k}^{(j)} /\left|\mathcal{I}^{k}\right|, & \text { if }(j, k) \in \mathcal{I}^{k} \\
0, & \text { otherwise },\end{cases} \\
\text { and } K_{k, j}:= \begin{cases}K_{k}^{(j)}, & \text { if }(j, k) \in \mathcal{I}^{k} \\
0, & \text { otherwise }\end{cases}
\end{gathered}
$$

The main result of this paper is the following:

Theorem 6. Assume that the transmission graph $\Gamma$ is composed of internally well-posed, impedance passive (conservative) strong boundary nodes $\Xi^{(j)}=\left(G^{(j)}, L^{(j)}, K^{(j)}\right)$ with the following property:

$$
\text { all of the operators }\left[\begin{array}{l}
G^{(j)} \\
K^{(j)}
\end{array}\right] \text { are surjective. }
$$

Then the colligation of $\Gamma$ is an impedance passive (resp., conservative), internally well-posed strong boundary node.

This is proved in three steps (Lemmas 7, 8, and 9) presented in the following section with sketches of their proofs. The assumption (10) can be relaxed but it appears to hold in many applications, as in our example.

\section{COUPLINGS BETWEEN COLLIGATIONS}

In this section, we present three elementary couplings between boundary control systems. We show that if one starts with internally well-posed, impedance passive boundary nodes, then the coupled systems are such as well. We remark that any transmission graph can be reconstructed by iterated application of these elementary couplings. Section 4.1 deals with a partial parallel coupling between two compatible colligations, see Fig. 2 (left). Such parallel couplings are treated in (Staffans, 2005, Examples 2.3.13 and 5.1.17) for system nodes. In Section 4.2, we form loops by joining two signals of a single colligation, see Fig. 2 (right). Both the control vertices and the closed vertices are treated similarly at this stage: all the vertices are left "open" so that (8) is satisfied but (9) is not. After

\footnotetext{
2 In sums of $\mathcal{U}$ and $\mathcal{Y}$, pick one pair $(j, i) \in \mathcal{I}^{k}$ for each $k$.
}
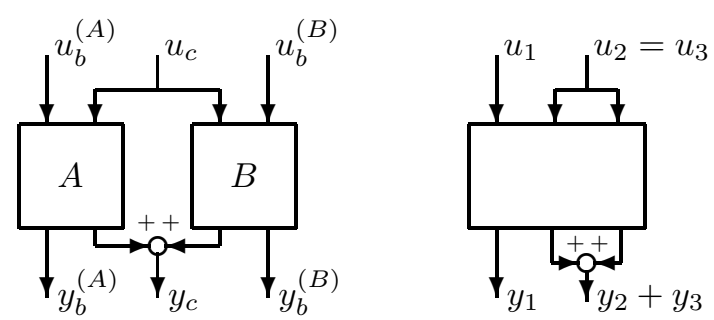

Fig. 2. Left: Partial parallel coupling; Right: Loop coupling constructing the full coupling graph structure by making a finite number of parallel and loop couplings, the final step - presented in Section 4.3 - is taken to close those vertices that are not used for control/observation; then condition (9) is satisfied, too. The transmission graph $\Gamma$ and its colligation has now been reconstructed, and the remaining (open) vertices are exactly the control vertices of $\Gamma$. Note that property (10) is preserved in parallel and loop couplings and it is only needed when closing the vertices.

\subsection{Partial parallel coupling}

Assume that $\Xi^{(j)}=\left(\left[\begin{array}{l}G_{b}^{(j)} \\ G_{c}^{(j)}\end{array}\right], L^{(j)},\left[\begin{array}{c}K_{b}^{(j)} \\ K_{c}^{(j)}\end{array}\right]\right), j=A, B$ are colligations on the Hilbert spaces $\left(\left[\begin{array}{c}\mathcal{U}_{b}^{(j)} \\ \mathcal{U}_{c}\end{array}\right], \mathcal{X}^{(j)},\left[\begin{array}{c}\mathcal{Y}_{b}^{(j)} \\ \mathcal{Y}_{c}\end{array}\right]\right)$ with solution spaces $\mathcal{Z}^{(j)}$. Define the composed colligation $\Xi^{(A B)}:=\left(G^{(A B)}, L^{(A B)}, K^{(A B)}\right)$ on the Hilbert spaces $\left(\mathcal{U}^{(A B)}, \mathcal{X}^{(A B)}, \mathcal{Y}^{(A B)}\right)$ where $\mathcal{U}^{(A B)}:=\mathcal{U}_{b}^{(A)} \oplus \mathcal{U}_{c} \oplus \mathcal{U}_{b}^{(B)}$, $\mathcal{X}^{(A B)}:=\mathcal{X}^{(A)} \oplus \mathcal{X}^{(B)}$, and $\mathcal{Y}^{(A B)}:=\mathcal{Y}_{b}^{(A)} \oplus \mathcal{Y}_{c} \oplus \mathcal{Y}_{b}^{(B)}$ by

$$
\begin{aligned}
G^{(A B)}:= & {\left[\begin{array}{cc}
G_{b}^{(A)} & 0 \\
G_{c}^{(A)} & 0 \\
0 & G_{b}^{(B)}
\end{array}\right], \quad L^{(A B)}:=\left[\begin{array}{cc}
L^{(A)} & 0 \\
0 & L^{(B)}
\end{array}\right], } \\
& \text { and } K^{(A B)}:=\left[\begin{array}{cc}
K_{b}^{(A)} & 0 \\
K_{c}^{(A)} & K_{c}^{(B)} \\
0 & K_{b}^{(B)}
\end{array}\right] .
\end{aligned}
$$

The domain of the colligation is

$$
\operatorname{dom}\left(\Xi^{(A B)}\right):=\left[\begin{array}{l}
\operatorname{dom}\left(\Xi^{(A)}\right) \\
\operatorname{dom}\left(\Xi^{(B)}\right)
\end{array}\right] \cap \mathcal{N}\left(\left[\begin{array}{ll}
G_{c}^{(A)} & -G_{c}^{(B)}
\end{array}\right]\right) .
$$

Such partial parallel coupling is illustrated in Fig. 2 (left). Lemma 7. Let $\Xi^{(A)}, \Xi^{(B)}$, and $\Xi^{(A B)}$ be as defined above. If the colligations $\Xi^{(A)}$ and $\Xi^{(B)}$ are internally well-posed, impedance passive (conservative) strong boundary nodes such that both $\left[\begin{array}{c}G^{(A)} \\ K^{(A)}\end{array}\right]$ and $\left[\begin{array}{c}G^{(B)} \\ K^{(B)}\end{array}\right]$ are surjective, then the composed colligation $\Xi^{(A B)}$ is an internally well-posed, impedance passive (resp., conservative) strong boundary node with the property that $\left[\begin{array}{c}G^{(A B)} \\ K^{(A B)}\end{array}\right]$ is surjective.

Proof. This can be proved by first showing that $\Xi^{(A B)}$ is a strong colligation. Here it is helpful to note that strongness of $\Xi^{(j)}, j=A, B$ implies that $G^{(j)}$ and $K^{(j)}$ are continuous with respect to the graph norm of $L^{(j)}$ by (Malinen and Staffans, 2007, Lemma 4.5).

Then the desired result can be obtained using (Malinen and Staffans, 2007, Theorem 4.3 and Remark 4.6). 


\subsection{Loop coupling}

Assume that $\Xi=(G, L, K)$ is a colligation on the Hilbert spaces $\left(\left[\begin{array}{l}\mathcal{U}_{1} \\ \mathcal{U}_{c} \\ \mathcal{U}_{c}\end{array}\right], \mathcal{X},\left[\begin{array}{l}\mathcal{Y}_{1} \\ \mathcal{Y}_{c} \\ \mathcal{Y}_{c}\end{array}\right]\right)$ where $G=\left[\begin{array}{lll}G_{1} & G_{2} & G_{3}\end{array}\right]^{T}$ and $K=\left[\begin{array}{lll}K_{1} & K_{2} & K_{3}\end{array}\right]^{T}$. Two of these parts are "glued" together to form another colligation $\widehat{\Xi}:=(\widehat{G}, \widehat{L}, \widehat{K})$ on the Hilbert spaces $\left(\left[\begin{array}{l}\mathcal{U}_{1} \\ \mathcal{U}_{c}\end{array}\right], \mathcal{X},\left[\begin{array}{l}\mathcal{Y}_{1} \\ \mathcal{Y}_{c}\end{array}\right]\right)$ with $\operatorname{dom}(\widehat{\Xi}):=$ $\left\{z \in \operatorname{dom}(\Xi) \mid G_{2} z=G_{3} z\right\}, \widehat{G}:=\left[\begin{array}{l}G_{1} \\ G_{2}\end{array}\right], \widehat{L}:=\left.L\right|_{\operatorname{dom}(\widehat{\Xi})}$, and $\widehat{K}:=\left[\begin{array}{c}K_{1} \\ K_{2}+K_{3}\end{array}\right]$.

The block diagram of such coupling is shown on the right in Fig. 2. As with the parallel coupling, we note that the coupling preserves all the desired properties:

Lemma 8. Let $\Xi$ and $\widehat{\Xi}$ be as defined above. If the colligation $\Xi$ is an internally well-posed, impedance passive (conservative) strong boundary node such that $\left[\begin{array}{ll}G & K\end{array}\right]^{T}$ is surjective, then also $\widehat{\Xi}$ is an internally well-posed, impedance passive (resp., conservative) strong boundary node with the property that $\left[\begin{array}{ll}\widehat{G} & \widehat{K}\end{array}\right]^{T}$ is surjective.

The proof is very similar to that of Lemma 7 .

\subsection{Closing the vertices}

In this step, we single out the control/observation vertices and permanently "close" all others with respect to additional external signals. Note that after the parallel and loop couplings, under the assumptions of Lemmas 7 and 8 , the resulting colligation is an internally well-posed boundary node, such that condition (i) of Definition 4 is satisfied. This closing means that also the condition (ii) of Definition 4 is required. This can be done without sacrificing the internal well-posedness or passivity/conservativity.

So let $\Xi=(G, L, K)$ be a colligation on the Hilbert spaces $\left(\left[\begin{array}{l}\mathcal{U}_{1} \\ \mathcal{U}_{2}\end{array}\right], \mathcal{X},\left[\begin{array}{l}\mathcal{Y}_{1} \\ \mathcal{Y}_{2}\end{array}\right]\right)$ with splittings $G=\left[\begin{array}{l}G_{1} \\ G_{2}\end{array}\right]$ and $K=\left[\begin{array}{c}K_{1} \\ K_{2}\end{array}\right]$ where $G_{2}$ and $K_{2}$ correspond to vertices that will be closed. Define the new colligation by $\widehat{\Xi}:=\left(G_{1}, \widehat{L}, K_{1}\right)$ on the Hilbert spaces $\left(\mathcal{U}_{1}, \mathcal{X}, \mathcal{Y}_{1}\right)$ with $\operatorname{dom}(\widehat{\Xi}):=\operatorname{dom}(\Xi) \cap$ $\mathcal{N}\left(K_{2}\right)$ and $\widehat{L}:=\left.L\right|_{\operatorname{dom}(\widehat{\Xi})}$.

Lemma 9. Let $\Xi$ and $\widehat{\Xi}$ be as defined above. If $\Xi$ is an internally well-posed, impedance passive (conservative) strong boundary node with the property that $\left[\begin{array}{c}G \\ K\end{array}\right]$ is surjective, then also $\widehat{\Xi}$ is an internally well-posed, impedance passive (resp., conservative) strong boundary node.

Proof. We carry out a partial flow inversion and interchange the roles of $G_{2}$ and $K_{2}$. More precisely, it is proved that $\widetilde{\Xi}:=(\widetilde{G}, L, \widetilde{K})$ on Hilbert spaces $\left(\left[\begin{array}{l}\mathcal{U}_{1} \\ \mathcal{Y}_{2}\end{array}\right], \mathcal{X},\left[\begin{array}{l}\mathcal{Y}_{1} \\ \mathcal{U}_{2}\end{array}\right]\right)$ where $\widetilde{G}:=\left[\begin{array}{l}G_{1} \\ K_{2}\end{array}\right], \widetilde{K}:=\left[\begin{array}{l}K_{1} \\ G_{2}\end{array}\right]$, and $\operatorname{dom}(\widetilde{\Xi}):=\operatorname{dom}(\Xi)$, is an internally well-posed, impedance passive (conservative) strong boundary node. Colligation $\widehat{\Xi}$ is then obtained from $\widetilde{\Xi}$ by restricting the solution space to $\mathcal{N}\left(K_{2}\right)$, and it clearly has all the properties as claimed, see Definition 2 and (Malinen and Staffans, 2007, Lemma 4.5) concerning the strongness of $\widehat{\Xi}$.
It is trivial that $\widetilde{\Xi}$ is a strong colligation. One way to see the interchangeability of $G_{2}$ and $K_{2}$ is directly by Definition 3. Finally, the internal well-posedness can be shown with the help of (Malinen and Staffans, 2007, Theorem 4.7).

\subsection{Webster's equation on a graph}

Let us return to the example presented in the introduction. There we have four boundary nodes $\Xi^{(j)}, j=A, \ldots, D$ whose input and output spaces are split into two parts specified in (4), that is, $k_{j}=2$. The vertices are labelled with 1 and 2 and the arrows in Figs. 1 and 3 point from 1 to 2 . Thus, the index set is

$$
\text { Ind }=\{(j, i) \mid j=A, \ldots, D, i=1,2\} .
$$

Now the dynamical system given by (1), (2), and (3) corresponds to the colligation of the transmission graph $\Gamma:=$ $\left(\left\{\Xi^{(j)}\right\}_{j=A}^{D},\left\{\mathcal{I}^{1}\right\},\left\{\mathcal{J}^{l}\right\}_{l=1}^{2}\right)$ where the control vertex is $\mathcal{I}^{1}=\{(A, 1),(C, 1)\}$ and the two closed vertices are $\mathcal{J}^{1}=$ $\{(A, 2),(B, 1),(D, 2)\}$ and $\mathcal{J}^{2}=\{(B, 2),(C, 2),(D, 1)\}$. More precisely, equations (2) are equivalent with (8) and (9) and the input and output operators given in Definition 5 yield the input/output of equation (3).

Next we reconstruct this transmission graph in four phases which are illustrated in Fig. 3 .

\section{- Phase 1}

We begin with colligations $\Xi^{(j)}=\left(\left[\begin{array}{c}G_{1}^{(j)} \\ G_{2}^{(j)}\end{array}\right], L^{(j)},\left[\begin{array}{c}K_{1}^{(j)} \\ K_{2}^{(j)}\end{array}\right]\right)$ on Hilbert spaces $\left(\left[\begin{array}{l}\mathcal{U}_{1}^{(j)} \\ \mathcal{U}_{2}^{(j)}\end{array}\right], \mathcal{X}^{(j)},\left[\begin{array}{l}\mathcal{Y}_{1}^{(j)} \\ \mathcal{Y}_{2}^{(j)}\end{array}\right]\right), j=A, B, C, D$.

\section{- Phase 2}

The system $A$ is connected to $B$, and $C$ to $D$, by a partial parallel coupling so that we obtain two colligations $\Xi^{(A B)}$ and $\Xi^{(C D)}$ with

$$
\begin{array}{r}
G^{(A B)}=\left[\begin{array}{cc}
G_{1}^{(A)} & 0 \\
G_{2}^{(A)} & 0 \\
0 & G_{2}^{(B)}
\end{array}\right], \quad K^{(A B)}=\left[\begin{array}{cc}
K_{1}^{(A)} & 0 \\
K_{2}^{(A)} & K_{1}^{(B)} \\
0 & K_{2}^{(B)}
\end{array}\right], \\
\text { and } \operatorname{dom}\left(\Xi^{(A B)}\right)=\left\{\left[\begin{array}{l}
z^{(A)} \\
z^{(B)}
\end{array}\right] \in\left[\begin{array}{l}
\operatorname{dom}\left(\Xi^{(A)}\right) \\
\operatorname{dom}\left(\Xi^{(B)}\right)
\end{array}\right]\right. \\
\left.G_{2}^{(A)} z^{(A)}=G_{1}^{(B)} z^{(B)}\right\}
\end{array}
$$

and similarly $G^{(C D)}, K^{(C D)}$, and $\operatorname{dom}\left(\Xi^{(C D)}\right)$.

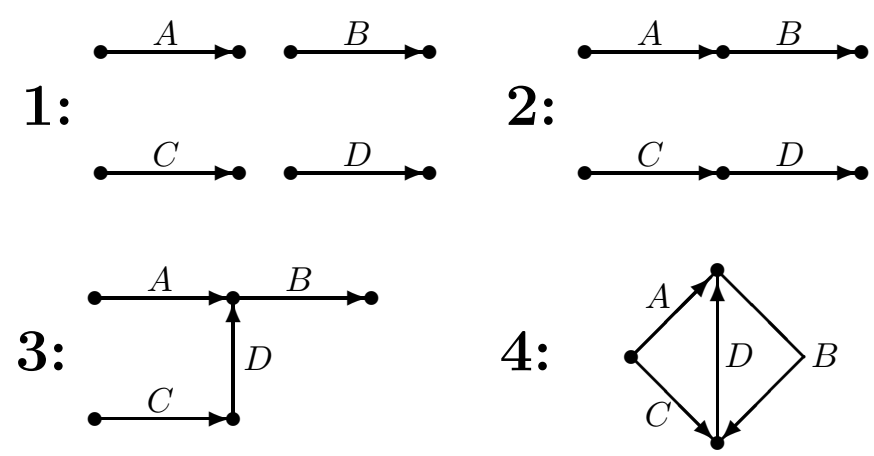

Fig. 3. Composing a transmission graph 


\section{- Phase 3}

Now $\Xi^{(A B)}$ is connected to $\Xi^{(C D)}$ by a partial parallel coupling. The part of the operator $G^{(A B)}$ which is not involved in the connection is $G_{b}^{(A B)}=\left[\begin{array}{cc}G_{1}^{(A)} & 0 \\ 0 & G_{2}^{(B)}\end{array}\right]$ and the part that is, is $G_{c}^{(A B)}=\left[\begin{array}{ll}G_{2}^{(A)} & 0\end{array}\right]$. Correspondingly $K_{b}^{(A B)}=$ $\left[\begin{array}{cc}K_{1}^{(A)} & 0 \\ 0 & K_{2}^{(B)}\end{array}\right]$ and $K_{c}^{(A B)}=\left[\begin{array}{ll}K_{2}^{(A)} & K_{1}^{(B)}\end{array}\right]$. The system $\Xi^{(C D)}$ is connected by its free vertex $\{(D, 2)\}$ to the common vertex $\{(A, 2),(B, 1)\}$ of $\Xi^{(A B)}$ so the $C D$ operators are chosen differently, namely $G_{b}^{(C D)}=\left[\begin{array}{cc}G_{1}^{(C)} & 0 \\ 0 & G_{1}^{(D)}\end{array}\right]$, $G_{c}^{(C D)}=\left[\begin{array}{ll}0 & G_{2}^{(D)}\end{array}\right], K_{b}^{(C D)}=\left[\begin{array}{cc}K_{1}^{(C)} & 0 \\ K_{2}^{(C)} & K_{1}^{(D)}\end{array}\right]$, and $K_{c}^{(C D)}=\left[\begin{array}{ll}0 & K_{2}^{(D)}\end{array}\right]$.

Thus, as described in Section 4.1, we obtain a system with

$$
\begin{gathered}
G=\left[\begin{array}{cc|cc}
G_{1}^{(A)} & 0 & 0 & 0 \\
0 & G_{2}^{(B)} & 0 & 0 \\
\hline G_{2}^{(A)} & 0 & 0 & 0 \\
\hline 0 & 0 & G_{1}^{(C)} & 0 \\
0 & 0 & 0 & G_{1}^{(D)}
\end{array}\right], \\
K=\left[\begin{array}{cc|cc}
K_{1}^{(A)} & 0 & 0 & 0 \\
0 & K_{2}^{(B)} & 0 & 0 \\
\hline K_{2}^{(A)} & K_{1}^{(B)} & 0 & K_{2}^{(D)} \\
\hline 0 & 0 & K_{1}^{(C)} & 0 \\
0 & 0 & K_{2}^{(C)} & K_{1}^{(D)}
\end{array}\right],
\end{gathered}
$$

and

$$
\begin{gathered}
\operatorname{dom}(\Xi)=\left\{z^{(j)} \in \operatorname{dom}\left(\Xi^{(j)}\right), j=A, B, C, D \mid\right. \\
\left.G_{2}^{(A)} z^{(A)}=G_{1}^{(B)} z^{(B)}=G_{2}^{(D)} z^{(D)}, G_{2}^{(C)} z^{(C)}=G_{1}^{(D)} z^{(D)}\right\} .
\end{gathered}
$$

\section{- Phase 4}

The vertex $\{(B, 2)\}$ is connected to $\{(C, 2),(D, 1)\}$, and $\{(A, 1)\}$ to $\{(C, 1)\}$, by a loop coupling. The parts of input and output that are not involved in the connection are $G_{1}=\left[\begin{array}{llll}G_{2}^{(A)} & 0 & 0 & 0\end{array}\right]$ and $K_{1}=\left[\begin{array}{llll}K_{2}^{(A)} & K_{1}^{(B)} & 0 & K_{2}^{(D)}\end{array}\right]$. The operators that are involved are $G_{2}=\left[\begin{array}{cccc}G_{1}^{(A)} & 0 & 0 & 0 \\ 0 & G_{2}^{(B)} & 0 & 0\end{array}\right]$, $K_{2}=\left[\begin{array}{cccc}K_{1}^{(A)} & 0 & 0 & 0 \\ 0 & K_{2}^{(B)} & 0 & 0\end{array}\right], G_{3}=\left[\begin{array}{cccc}0 & 0 & G_{1}^{(C)} & 0 \\ 0 & 0 & 0 & G_{1}^{(D)}\end{array}\right]$, and $K_{3}=$ $\left[\begin{array}{cccc}0 & 0 & K_{1}^{(C)} & 0 \\ 0 & 0 & K_{2}^{(C)} & K_{1}^{(D)}\end{array}\right]$. As described in Section 4.2, the new input and output operators are $G=\left[\begin{array}{l}G_{1} \\ G_{2}\end{array}\right]$ and $K=\left[\begin{array}{c}K_{1} \\ K_{2}+K_{3}\end{array}\right]$. To $\operatorname{dom}(\Xi)$ we impose the additional condition $G_{2} z_{2}=G_{3} z_{3}$. In terms of the original blocks, this can be written as $G_{1}^{(A)} z^{(A)}=G_{1}^{(C)} z^{(C)}$ and $G_{2}^{(B)} z^{(B)}=G_{1}^{(D)} z^{(D)}$.

Finally, we close all but the vertex $\{(A, 1),(C, 1)\}$.

The example system has now been constructed using the three elementary couplings. We have shown above that these couplings preserve the internal well-posedness and impedance passivity/conservativity of systems. Now since
Webster's equation on a simple line defines an internally well-posed, impedance conservative BCS, we finally conclude that also the system defined on a network defines an internally well-posed, impedance conservative BCS.

\section{CONCLUSIONS}

Many kinds of passive BCSs from electrodynamics, acoustics, and mechanics can be interconnected with each other so that the resulting system is passive and well-posed. The controllability, observability, and strong stability of such systems can then be established by the well known techniques for passive systems. Strong stabilisation, optimal control, and design problems on transmission graphs can be approached using these methods. Also finite dimensional dynamical couplings can be introduced as in Weiss and Zhao (2009), but then the composed system is not purely of boundary control type.

\section{REFERENCES}

Aalto, A. and Malinen, J. (2010). Cauchy problems from networks of passive boundary control systems. Technical report, A594, Aalto University School of Science and Technology, Institute of Mathematics.

Cervera, J., van der Schaft, A.J., and Baños, A. (2007). Interconnection of port-Hamiltonian systems and composition of Dirac structures. Automatica (J. of IFAC), $43(2), 212-225$.

Derkach, V., Hassi, S., Malamud, M., and de Snoo, H. (2006). Boundary relations and their Weyl families. Transactions of the American Mathematical Society, 358(12), 5351-5400.

Fattorini, H.O. (1968). Boundary control systems. SIAM Journal of Control, 6(3), 349-385.

Gorbachuk, V.I. and Gorbachuk, M.L. (1991). Boundary Value Problems for Operator Differential Equations. Kluwer Academic Publishers.

Hannukainen, A., Lukkari, T., Malinen, J., and Palo, P. (2007). Vowel formants from the wave equation. Journal of Acoustical Society of America Express Letters, 122(1).

Kurula, M., Zwart, H., van der Schaft, A.J., and Behrndt, J. (2010). Dirac structures and their composition on Hilbert spaces. Journal of Mathematical Analysis and Applications, 372(2), 402-422.

Malinen, J. and Staffans O.J. (2006). Conservative boundary control systems. Journal of Differential Equations, 231(1), 290-312.

Malinen, J. and Staffans O.J. (2007). Impedance passive and conservative boundary control systems. Complex Analysis and Operator Theory, 1, 279-300.

Mei, C. and Mace, B.R. (2005). Wave reflection and transmission in Timoshenko beams and wave analysis of Timoshenko beam structures. Journal of Vibration and Acoustics, 127(4), 382-394.

Staffans, O.J. (2005). Well-Posed Linear Systems. Cambridge University Press.

Villegas, J. (2007). A Port-Hamiltonian Approach to Distributed Parameter Systems. Ph.D. thesis, University of Twente.

Weiss, G. (1994). Regular linear systems with feedback. Mathematics of Control, Signals, and Systems, 7, 23-57.

Weiss, G. and Zhao, X. (2009). Well-Posedness and controllability of a class of coupled linear systems. SIAM Journal of Control and Optimization, 48, 2719-2750. 